\title{
(1) \\ Radiodiagnosis \\ TELERADIOLOGY ADOPTION DURING COVID-19 PANDEMIC: A RADIOLOGIST'S PERSPECTIVE
}

\section{Dr Mayur Pankhania*}

DMRD, DNB, EDiR, CIIP / Sahyog Imaging Center, Department of Radiodiagnosis, PDU Medical College \& Government Hospital, Rajkot, Gujarat, India.

ABSTRACT In Mach 2020, WHO christened the highly infectious disease caused by novel Coronavirus (2019 - nCoV or SARS-CoV-2) that emerged in the city of Wuhan in Hubei province, China, as COVID-19 and declared it as a pandemic. In the following weeks, the disease has swept rapidly across most of the countries of the world. With the global health emergency that we are currently facing, Teleradiology adoption increased tremendously amongst the radiologists, who are playing the cruciate role in combating this deadly disease by imaging and predicting early diagnosis. As the pandemic has brought new challenges that necessitate radiologist to adopt routine off-site reporting, this allows the continuity of imaging services to the patients without sacrificing the quality of care. It also helps to maintain their workloads from a remote location and diminish radiologist exposure to infections. In this article, the authors review examples of current scope and applications of teleradiology practice in pandemic and beyond. In addition, the future impact and natural extension of these techniques in radiology practice are discussed.

\section{KEYWORDS : Radiology, COVID-19, Pandemic, Teleradiology}

INTRODUCTION

Teleradiology is headway in radiology that has encouraged medicinal services and spared numerous lives. Innovation has brought a change in outlook in the field of radiology and medicinal services all in all. When innovation is improving lives, teleradiology-a digital marvel is concentrating on improving patient consideration and experience. At the point when critical reports like MRI, CT Scan, X-ray, and so forth should be deciphered earnestly and a specialist probably won't be accessible, it can postpone treatment costing the patient's life.[1] Here teleradiology has a noteworthy task to carry out.

As COVID-19 keeps on spreading over the United States, social insurance staff are working all day, every day to treat it more than some other nation on the planet. As these experts keep on rewarding contaminated patients, they chance contracting and spreading the infection themselves. Presently like never before, deciding how to best ensure patients and office work force is basic. Telehealth by and large picked up energy when Medicare suspended limitations to getting to telehealth, permitting radiology to arrive at a more extensive populace from home all through the pandemic.[2]

Luckily, headways in clinical innovation permit some industry experts to work remotely, joining numerous Americans in staying away from infection presentation and endeavoring to "straighten the bend." Radiologists who have just been instrumental in the recognition and early analyses of COVID19 cases can viably lead their work from home with the utilization of teleradiology. Teleradiology gives indistinguishable usefulness to doctors from their on location workstations, permitting radiologists to understand pictures and analyze from the security of their home. As the battle against COVID-19 proceeds, there is a developing need to make teleradiology arrangements progressively normal.[3]

\section{TELERADIOLOGY AND ITS IMPACT ON COVID-19}

Teleradiology falls under the telehealth umbrella since radiologists can decipher pictures and speak with doctors electronically while working remotely. For instance, cloudbased workstations and portable applications permit radiologists and doctors to get to analyses, reports, and pictures regardless of their area by means of pictorial filing and correspondence frameworks (PACS).[4] Several outputs can be investigated and announced in as meager as 8 minutes utilizing this innovation, outperforming the business normal of 30 minutes. This permits the two radiologists and doctors to give capable and amazing consideration to the patient while lessening the danger of spreading or contracting COVID-19.

Also, the business has just become a generally advanced workforce. Numerous radiologists have started to see pictures at home, direct notes with voice acknowledgment and approve reports electronically. The first U.S. understanding with lab-affirmed illness was analyzed, to some degree, with chest radiography and the progressing treatment routine incorporates extra chest radiography.[5] As cases deluge, COVID-19 case pictures can be investigated remotely through teleradiology to help decide courses of treatment for contaminated cases.

The symptomatic is the center of current age clinical treatment. Teleradiology arrangement in India will be the essential piece of remote treatment methodology unavoidably, even post COVID-19 pandemic settlement. Re-appropriating Xray announcing on the web will be the standard.

Teleradiology announcing helps the emergency clinics and analytic focuses to get radiology reports from the certified and experienced radiologists expeditiously without employing them fulltime. On the opposite side, radiologists also have more chances to offer their types of assistance online to more customers. RSP (Radiology Service Providers), a cloud-based advanced stage planned by Universal Mednet, makes the teleradiology announcing administrations progressively reliable and future-arranged.[6]

\section{THE IMPORTANCE OF RADIOLOGY IN CORONAVIRUS PANDEMIC}

Radiology has been seen as of noteworthy incentive under the watchful eye of patients with Coronavirus. It has been seen that radiologic imaging findings and patterns seen on X-Ray and High Resolution computer tomography (HRCT) of chest are of distinctive for COVID-19 Disease. In China at the beginning phases of the scourge episode, before swab testing was promptly accessible, HRCT imaging was utilized by specialists to analysis the likelihood of COVID-19 disease, and it has likewise been utilized for assessing the severity, progression and follow-up. Hence the capacity to rapidly report examines led on patients associated to have COVID disease is with specific significance right now.[6,7]

\section{HOW DOES TELERADIOLOGY HELP?}

In the crisis setting, teleradiology guarantees that when an 
output is performed, it tends to be accounted for quickly and treatment choices might be made immediately.

In India we have an unbalanced number of radiologists situated in metros, with relative under-portrayal in level 2 urban areas and remote pieces of the nation. Thusly, in any event, when the imaging gear might be accessible, the radiologist may not be nearby at the hour of the x-ray or sweep to report it. Teleradiology is of extraordinary incentive in this condition. Our announcing of $\mathrm{x}$-rays performed at Community Health Centres over the province of Tripura by our national board of radiologists, is a case of such a situation. In a domain, for example, the current COVID pandemic where enormous quantities of chest Xrays and CT checks are probably going to being performed over a brief timeframe outline, teleradiology can be an important reinforcement.[8]

Further, in the course of recent decades, teleradiology has expanded universally. In our association a solitary radiologist can be announcing for various medical clinics over the globe at the same time, expanding the range and commitment of the radiologist a long ways past their revealing room. In the setting of a worldwide pandemic, having a board of radiologists spread over the globe who can give $24 \times 7$ inclusion is an important reinforcement for neighbourhood frameworks that might be overpowered during a local spike in cases. Particularly during the night move when staffing is short and staff are exhausted the accessibility of a reinforcement radiologist who is in a day-time region over the world can be of significant advantage. $[9,10]$

\section{EXPANSION OF TELERADIOLOGY DURING COVID-19}

The government perceived the job telehealth innovation could have during the coronavirus pandemic, since the Centres for Medicare and Medicaid Services declared a guideline that has expanded access to Medicare telehealth administrations. It permits recipients to get a more extensive scope of administrations from their PCPs without heading out to a medicinal services office. This guideline upgrades persistent consideration just as decreases the flood of individuals at physical clinical offices to limit further COVID-19 spread. Furthermore, there's a transitory unwinding of compulsory instate permitting necessity for radiologists that permits crossstate perusing with teleradiology. [11]

For radiologists, this presents a chance to grow their teleradiology innovation and accumulate volumes, which has been difficult to find as of late. Beforehand, routine X-beams in outpatient settings and radiological volumes have diminished as more Americans follow stay-at-home requests.[12] Notwithstanding, increasingly radiological administrations will presently be required at offices the country over in recognizing and rewarding COVID-19 cases. Executing teleradiology into these offices will permit more peruses to be handled and offer faster report turnarounds and better discoveries of the infection.[13] This can have a priceless effect in distinguishing COVID-19.

\section{BENEFITS OF TELERADIOLOGY IN THE CORONAVIRUS PANDEMIC}

In an unprecedented global situation such as the COVID-19 pandemic has created, teleradiology has shown that it affords unique benefits [Figure 1]:

\section{Allowing radiologists to work from home while maintaining} social distancing \& saftey

Radiology is a significant piece of the administration worldview for COVID-19, particularly given co-morbidities, concentrated consideration, and subsequent follow-up imaging. Hence, having radiologists consistently accessible to report filters is of basic significance. In this specific circumstance, teleradiology permits radiologists to work securely in/from their individual homes. It permits radiologists to stay disengaged from the remainder of their locale, which in this period of social separating assists with limiting infection transmission.[14] What's more, in the setting of isolate/detachment teleradiology permits radiologists to keep on being clinically profitable and bolster their clinical associates in tolerant consideration. While a few radiologists should be available at the medical clinic to perform nearby techniques, the lion's share who are in the interpretive job can report cases from offsite.[15]

\section{Ensuring 24/7 coverage}

In the course of recent decades, a tried and true advantage of teleradiology has been its capacity to traverse the globe, with a solitary radiologist detailing for various emergency clinics at the same time, expanding the span and commitment of the radiologist a long ways past their revealing room. In the setting of a worldwide pandemic, having a worldwide board of radiologists who between them can flawlessly give every minute of every day inclusion is a significant reinforcement for neighbourhood frameworks that might be overpowered during a territorial spike in cases.[16] So also, the night-to-day change that worldwide teleradiology bears gives a significant support to exhausted radiologists who are depleted, constraining their ability to cover a night move. During a pandemic-type circumstance, this extent of teleradiology is the thing that would render it of most extreme worth.

\section{Access to COVID-19 cases from around the globe}

Radiology has just assumed a critical job in the COVID-19 pandemic to date in different pieces of the world, including during the time span when testing was constrained or inaccessible. As the pandemic spreads over the globe, the capacity to separate imaging data from cases found in one piece of the world and use that experience to most extreme advantage in another part is a huge worth that worldwide teleradiology can give.

\section{Rapid online training and education}

Given its solid computerized imaging base, teleradiology takes into consideration the capacity to quickly make an interpretation of figuring out how to instructing and extend it to a worldwide crowd. Teleradiology permits a group of radiologists to remain for all intents and purposes associated both for consistent revealing work process, including conversation of complex cases, and for fast dispersal of continuous preparing refreshes and instructive exercises. An intermittent gathering meeting over an advanced/electronic conferencing stage can keep everybody associated for work process and quality-related conversations. For example, over our www.radguru.net gateway, we have just led a few instructional meetings on both clinical and radiologic boundaries of COVID-19 disease - both interior preparing for our own all inclusive spread gathering of radiologists and for the national and universal network of doctors and radiologists.[17]

PrOS
-Saftey of Radiologist \& staff during
pandemic
-Decrease workload on other radiologist
with fast service
-Availability of service $24 \times 7 \times 365$ days
- Solve radiologists shortage
- Remote rural area services
-A second opinion from an specialist
radiologist for difficult cases
-Improve recruiting of medical
professionals, give an educational chance
to expand their experience in different
regions
-Improving patient care, and prevent the
need for patients to travel to find medical
services or specialized experts.

\section{Cons}

-Expenses of installing a teleradiology system

-Teleradiology system maintenance -Technical issues \& Frequest System breakdowns -Legal jurisdiction of a teleradiology system

- Medical license

- Accreditation of teleradiology service - Limited access to obtain a previous studies and patients' history - Training staff and radiologist - Limited communication with clinicians. - Inadequat renumeration to radiologist

Figure 1: Pros \& Cons 
TELERADIOLOGY HAS HELPED US TO STAY AHEAD OF TIME DURING THIS PANDEMIC

The curve that is tossed at us as Covid-19 will urge a great deal of organizations and experts to work uniquely in contrast to they did pre-COVID. While there will be changes in the workplace working with advancements, for example, amazed timings being presented, remote working will be an immense change that will be executed over a ton of regions of work. One significant space that will undoubtedly embrace remote working is the 'radiology division' of indicative focuses and emergency clinics. The pattern is normally set to move towards online PACS frameworks in India. [18]

The expanded requirement for radiologists and the extraordinary lack of the specialists are going to make teleradiology one of the most reasonable answers for a colossal clinical issue. In the occasions to come, it is urgent for clinical foundations and indicative focuses to search for top PACS suppliers in India. Taking a gander at the current day clinical circumstance over the globe, it very well may be securely expected that teleradiology can hold a money box of advantages for the supporters that settle on the administrations. [18]

One of the most noteworthy advantages of radiology data frameworks is the simple access to specialists. At the point when radiologists the nation over are in a rush, a great deal of clinical offices think that its difficult to get hold of a specialist that can offer his types of assistance on schedule. With the assistance of online teleradiology stages, it is conceivable to approach the best radiologists without the limitations of time and separation keeping them down.

Being a piece of the social insurance field accompanies an extraordinary obligation of serving those out of luck, and this is something that might be in risk in the current occasions. With the assistance of remote radiology revealing administrations, it tends to be ensured that your clinical office is consistently open to its patients and that nobody should be dismissed essentially in light of the inaccessibility of radiology specialists. A noteworthy advantage of the teleradiology framework is that nobody needs to endure in light of the COVID - 19 circumstance that the world is battling with. Then again, the teleradiology programming suppliers in India can likewise help in ensuring that no business is lost for the need of specialists. $[2,17,18]$

While it is fundamental to serve patients, they can't be truly served if the clinical offices can't do new business. With the assistance of DICOM watchers and man-made consciousness in radiology, it is conceivable to remain in front of the occasions and acknowledge the changing period of clinical working emphatically.

\section{TELERADIOLOGY IMPACT BEYOND COVID-19}

While the overwhelming utilization of teleradiology has been incited by grievous conditions due to COVID-19, it might turn into the new business standard long after the pandemic is killed.[19] The expanded precision, quality and speed teleradiology brings-combined with improving work process-will provoke medical clinics to actualize these administrations all through their offices. Not exclusively does teleradiology improve by and large radiologist work process and patient consideration, yet it additionally diminishes nearby expenses.[20,21] This is something offices are continually hoping to do, particularly in the current money related catastrophe we are persevering.

Doctors will likewise observe a lot of progress in this change. All through the rest of the coronavirus pandemic, doctors will get acquainted with working and speaking with radiologists by means of these administrations. Teleradiology's capacities makes the cooperation between the two sides tremendously simpler while additionally improving doctors' general capability. Along these lines, we can foresee that it'll be hard to come back to past strategies once things come back to ordinary-another marker that teleradiology is the eventual fate of the business.[21]

Teleradiology administrations can turn into a significant answer for the COVID-19 emergency and past. Making these sorts of administrations progressively available to radiologists over the United States will have an enduring effect on the business. More peruses will be dissected and analyzed while permitting radiologists to maintain CDC precautionary measures in working remotely to diminish viral introduction. Teleradiology isn't the main answer for the COVID-19 emergency, yet it very well may be a significant advance as it turns out to be progressively regular all through our social insurance frameworks. [22,23]

\section{CONCLUSION}

The COVID-19 pandemic has brought new challenges that are necessitate radiologist to began use of Teleradiology and other virtual technology tools to maintain their workloads from a remote location, and decrease radiologist exposure to infections. Teleradiology reporting and adoption increased tremendously during this pandemic and is likely to continue. Although, it has some concerns regarding decreased in rapport, which was more common in private practices than academic hospital. As frontline working staff gets more used to teleradiology along with telemedicine in general, its becomes more acceptable to hospitals, referring physicians, radiologist and patients; and in future it's become more of a standard way of practicing medicine and may persist PostCOVID 19.

\section{REFERENCES}

1. Silva E 3rd, Breslau J, Barr RM, et al. ACR white paper on teleradiology practice: a report from the Task Force on Teleradiology Practice. J Am Coll Radiol 2013;10:575-85

2. Larson PA, Janower ML. The nighthawk: bird of paradise or albatross? J Am Coll Radiol 2005;2:967-70.

3. Centers for Disease Control and Prevention. Coronavirus disease (COVID19). Cases in the U.S. Available at: https://www.cdc.gov/coronavirus/2019ncov/cases-up-dates/cases-in-us.html. Accessed March 10, 2020.

4. Coronavirus disease 2019 (COVID-19). Atlanta: Centers for Disease Control and Prevention, https://www.cdc.gov/coronavirus/2019-nCoV/index.html

5. Ruan S. Likelihood of survival of coronavirus disease 2019. Lancet Infect Dis. 2020 Mar 30:S1473-3099(20)30257-7.

6. ACR Recommendations for the use of chest radiography and computed tomography (CT) for suspected COVID-19 infection. Reston, VA: American College of Radiology; 2020 March 11.

7. Simpson S, Kay FU, Abbara S, Bhalla S, Chung JH, Chung M, Henry TS, Kanne JP, Kligerman S, Ko JP, Litt H. Radiological Society of North America expert consensus statement on reporting chest CT findings related to COVID19. Endorsed by the Society of Thoracic Radiology, the American College of Radiology, and RSNA. Radiology: Cardiothoracic Imaging. 2020 Mar 25;2(2):e200152.

8. Ai T, Yang Z, Hou H, Zhan C, Chen C, Lv W, Tao Q, Sun Z, Xia L. Correlation of chest CT and RT-PCR testing in coronavirus disease 2019 (COVID-19) in China: a report of 1014 cases. Radiology. 2020 Feb 26:200642.

9. Emergency use authorization, ID NOW COVID-19, Abbott Diagnostics Scarborough Inc. Washington, DC: U.S. Food \& Drug Administration; 2020 March 27

10. PIH Guide COVID-19 Part II: Clinical management of COVID-19. Boston, MA Partners In Health; 2020 March 21

11. Biomerica starts shipping finger-prick blood test samples for COVID-19. Medicaldevice-network.com; 2020 March 18

12. Government of Canada. Coronavirus disease (COVID-19): out-break update. Accessed April 30, 2020.

13. Centers for Disease Control and Prevention. Coronavirus disease2019 (COVID-19) symptoms. 2020. Accessed April 30, 2020.

14. Center for Disease Control and Prevention. Coronavirus disease 2019 (COVID-19). Preventing getting sick. 2020. Accessed April 30, 2020.

15. The Lancet. Redefining vulnerability in the era of COVID-19. Lancet. 2020;395(10230):1089.

16. Boseley $\mathrm{S}$. Test and trace: lessons from Hong Kong on avoiding a coronavirus lockdown. The Guardian. April 17, 2020

17. Kooraki S, Hosseiny M, Myers L, Gholamrezanezhad A. Coronavirus (COVID19) outbreak: what the department of radiology should know. J Am Coll Radiol. 2020;17(4):447-451.

18. Canadian Association of Radiologists, Canadian Society of Interventional Radiology. Canadian Society of Interventional Radiology (CAIR) and Canadian Association of Radiologists (CAR) Statement on COVID-19. 2020. Updated March 24, 2020. Accessed April 30, 2020.

19. American College of Radiology. American College of Radiology Breast Imaging Reporting and Data System Atlas (BI-RADSAtlas). American College 
of Radiology; 2013.

20. Dalla Palma L., Stacul F. et al. Relationships between Radiologists and Clinicians: Results of three surveys. Clin. Radiol., 2000; 55: 602-605.

21. Board of the Faculty of Clinical Radiology. To err is human: The case for review of reporting discrepancies. London: Royal College of Radiologists, 2001; BFCR (01) 4

22. Chandy J., Goodfellow T., Vohrah A. Clinical governance in action: radiology. Hosp Med, 2000; 61: 326-329. 